\title{
Estudio randomizado multicéntrico de cerclaje para pre- vención del parto prematuro en pacientes de alto riesgo y cuello corto en el trimestre medio. (1)
}

\author{
Owen J, Hankins G, lams JD, Berghella V, Sheffield JS, Perez-Delboy A, Egerman RS, \\ Wing DA, Tomlinson M, Silver R, Ramin SM, Guzman ER, Gordon M, How HY, Knudt- \\ son EJ, Szychowski JM, Cliver S, Hauth JC. Am J Obstet Gynecol 2009;201:375.e1-8. \\ Análisis crítico: María Isabel Sigüenza V. ${ }^{1}$, Claudio Vera PG. ${ }^{2,3} M S c$, Jorge Carvajal C. ${ }^{2}$ PhD \\ 1 Posgrado de Obstetricia y Ginecología, ${ }^{2}$ Departamento de Obstetricia y Ginecología, Unidad de Medicina Materno \\ Fetal, ${ }^{3}$ Unidad de Medicina Basada en Evidencias, Facultad de Medicina, Pontificia Universidad Católica de Chile.
}

\section{RESUMEN (1)}

Objetivo: El objetivo del estudio fue probar el cerclaje para prevenir el parto prematuro recurrente en mujeres con cuello corto. Método: Mujeres con antecedente de parto prematuro espontáneo menor a 34 semanas fueron tamizadas para cuello corto y asignadas al azar a cerclaje si la longitud cervical era menor a 25 mm. Resultados: De 1014 mujeres tamizadas, 302 fueron randomizadas; $42 \%$ de las mujeres no asignadas y $32 \%$ de las asignadas a cerclaje tuvieron un parto antes de las 35 semanas $(p=0,09)$. En el análisis planeado, parto antes de las 24 semanas $(p=0,03)$ y mortalidad perinatal $(p=0,46)$ fueron menos frecuentes en el grupo cerclaje. Hubo interacción significativa entre longitud cervical y cerclaje. Parto antes de las 35 semanas $(p=0,006)$ fue reducido en el estrato menor a 15 $\mathrm{mm}$, con efecto nulo en el estrato 15-24 mm. Conclusión: En mujeres con un parto prematuro espontáneo previo antes de las 34 semanas y longitud cervical menor a $25 \mathrm{~mm}$, el cerclaje reduce el parto en período previable y la mortalidad perinatal, pero no previene parto prematuro menor a 35 semanas, a menos que la longitud cervical sea menor a 15 $\mathrm{mm}$.

\section{ANÁLISIS DE LA INVESTIGACIÓN}

\section{A. Relevancia clínica de la investigación}

La pregunta: ¿en pacientes con alto riesgo de parto prematuro (antecedentes de parto $<34$ semanas) y cuello corto $(<25 \mathrm{~mm})$, el cerclaje disminuye el riesgo de parto prematuro (antes de las 35 semanas)? Escenario clínico: El parto prematuro, junto a las malformaciones congénitas, son las dos causas más frecuentes de morbimortalidad perinatal. Estudios anteriores han revelado que el riesgo de parto prematuro es inversamente proporcional a la longitud del canal cervical (2), con medición efectuada de modo confiable y reproducible con ultrasonido transvaginal (3). Se define como población de riesgo a las personas que tienen un canal cervical bajo el percentil 10, lo que equivale a $25 \mathrm{~mm}$ (4). El mejor manejo para pacientes con riesgo elevado de parto prematuro y cuello corto no ha sido establecido, siendo el cerclaje una alternativa evaluada en estudios clínicos, con resultados variables.

\section{B. El estudio (1)}

Originalidad del estudio: Existe evidencia insufi- 
ciente, para determinar el balance riesgo-beneficio del cerclaje, en pacientes con antecedentes de parto prematuro y cuello corto. El estudio analizado aportará evidencia relevante a un tema no resuelto. Población estudiada: Mujeres con embarazo único entre las $16+6$ y $21+6$ semanas de gestación, en control por antecedente de parto prematuro, con detección de canal cervical menor a $25 \mathrm{~mm}$ en el tamizaje. Criterios de exclusión: Malformaciones fetales, cerclaje electivo por historia de insuficiencia cervical, complicaciones materno fetales significativas (inmunización $\mathrm{RH}$, hipertensión crónica, diabetes insulino dependiente), canal cervical mayor a $25 \mathrm{~mm}$ a las 22 semanas de gestación. Intervención: Las pacientes se asignaron al azar a cerclaje vs no cerclaje. Se utilizó un método de aleatorización computacional por bloques permutados y se estratificó por: centro, longitud cervical y uso de progesterona. El seguimiento pos randomización fue igual en ambos grupos de pacientes. El cerclaje fue con técnica de McDonald, el uso de antibióticos y tocolíticos no se incluyó en el protocolo y quedó a discreción de cada centro. El cerclaje fue retirado a las 37 semanas si las pacientes no iniciaron trabajo de parto. Resultados: Se identificó un total de 1044 pacientes con alto riesgo de parto prematuro; 1014 (99\%) firmaron el consentimiento para seguimiento ecográfico del canal cervical, solo en $831(82 \%)$ se confirmó el antecedente de parto prematuro al revisar las fichas clínicas; en 318 (31\%) se encontró el cuello $<25 \mathrm{~mm}$; 16 pacientes no dieron el consentimiento para ingresar al estudio. Un total de $302(95 \%)$ fueron randomizadas, 153 al grupo no cerclaje y 148 al grupo cerclaje. Del grupo no cerclaje, en 14 pacientes se realizó la intervención (10 por insuficiencia cervical; 4 por recomendación de sus tratantes). Del grupo cerclaje, en 11 pacientes no se realizó la intervención (8 rechazaron el procedimiento y 3 por infección intraamniotica, muerte fetal o cervicitis). Resultados perinatales: No hubo diferencias entre el grupo cerclaje y el grupo control en cuanto al evento primario: parto antes de las 35 semanas. Entre los resultados secundarios se observó reducción de la tasa de parto antes de la viabilidad, reducción de la mortalidad perinatal y del riesgo de parto antes de las 35 semanas en pacientes con cuello menor a $15 \mathrm{~mm}$ (Tabla 1).

\section{Análisis crítico}

Validez interna: El diseño del estudio es apropiado a la pregunta que origina la investigación, hubo cálculo apropiado del tamaño muestral, la randomización (por bloques y estratificada) fue bien efectuada, con ocultamiento de la secuencia de randomización. Los objetivos primarios y secundarios están claramente definidos. El análisis de los datos se describe de acuerdo al principio de intención de tratar. No hubo doble ciego por las características propias de la intervención, pero el resultado medido (parto antes de las 35 semanas) es objetivo, por lo que esto no debiera ser un problema. Se especifica que no hubo co-intervenciones (las mujeres de ambos grupos tuvieron el mismo cuidado prenatal). Comentario: El estudio analizado es de buen diseño e implementación y presenta bajo riesgo de sesgo. Permite concluir que el cerclaje en mujeres de riesgo de parto prematuro, con cuello menor a $25 \mathrm{~mm}$, no reduce el riesgo de parto prematuro menor a 35 semanas. El análisis planeado de resultados secundarios sugiere que en pacientes con canal cervical menor a $15 \mathrm{~mm}$, el cerclaje si reduce el riesgo de parto prematuro ( $<35$ semanas). Dado que el tamaño muestral no fue calculado para este análisis, no permite establecer esta como una conclusión válida del estudio, pero si sugiere la necesidad de un nuevo estudio que investigue específicamente esta posibilidad.

Tabla I

RESULTADOS DE LA INVESTIGACIÓN (1)

\begin{tabular}{ccccc}
\hline & Cerclaje & No cerclaje & OR [95\% IC] & Valor $p$ \\
$(\%)$ & $(\%)$ & &
\end{tabular}

Resultado primario:

Parto $<35$ sem

Resultados secundarios:

Parto $<24$ sem

Mortalidad perinatal

Parto $<35$ con cuello $<15 \mathrm{~mm}$

$\begin{array}{ll}6,1 & 14 \\ 8,8 & 16 \\ 14,3 & 42\end{array}$

$0,41[0,18-0,93]$

42




\section{REFERENCIAS}

1. Owen J, Hankins G, lams JD, Berghella V, Sheffield JS, Perez-Delboy A, et al. Multicenter randomized trial of cerclage for preterm birth prevention in high-risk women with shortened midtrimester cervical length. Am J Obstet Gynecol 2009;201:375.e1-8.

2. To MS, Skentou CA, Royston P, Yu CK, Nicolaides $\mathrm{KH}$. Prediction of patient-specific risk of early preterm delivery using maternal history and sonogra- phic measurement of cervical length: a populationbased prospective study. Ultrasound Obstet Gynecol 2006;27:362-7.

3. Bergella V. The short and funneled cervix: what do I do now? Contemp Ob/Gyn 2004;49:26-34.

4. lams JD, Goldenberg RL, Meis PJ, Mercer BM, Moawad A, Das A, et al. The length of the cervix and the risk of spontaneous preterm delivery. $\mathrm{N}$ Engl $\mathrm{J}$ Med 1996;334:567-72. 